\title{
Política de Agrupamiento Escolar: ¿Mutación o "muerte" del trabajo de Director Escolar?
}

\author{
María Cecilia Bocchio ${ }^{\mathrm{i}}$ \\ Universidad Nacional de Córdoba, Argentina
}

\begin{abstract}
Resumen
La política de Agrupamiento Escolar (AE) definió un nuevo modelo normativo de organización escolar en Portugal. A través de este artículo, enmarcado en una tesis doctoral, procuramos dar cuenta de (a) el proceso histórico hasta la configuración actual de la política de AE. Seguidamente, abordamos (b) las implicancias del AE como política que, discursivamente, pretende promover la descentralización del Sistema Educativo portugués. Posteriormente, presentamos (c) los principales ejes teórico-metodológicos del estudio de caso desarrollado en un AE perteneciente a la Dirección Regional de Lisboa y Valle del Tejo y (d) algunos resultados obtenidos en la investigación. Finalmente, planteamos (e) algunos de los principales avances interpretativos acerca de las transformaciones en la dirección del ficticiamente denominado "AE Margen" y concretamente en el trabajo de su Director, una figura que ya no va a volver a coordinar y dirigir sólo una escuela.
\end{abstract}

Palabras-clave

Políticas educativas; Agrupamiento Escolar; Director de Agrupamiento Escolar

\section{Introducción}

La política de Agrupamiento Escolar (AE) nace como una iniciativa para responder a la necesidad de reordenar la administración escolar portuguesa, marcada por la existencia de una gran cantidad de escuelas de enseñanza básica con matrículas muy reducidas (Afonso, 2005). En la 
investigación desarrollada ${ }^{1}$ se aborda el trabajo del Director de un "Agrupamento de Escolas" como actor regulado por el Ministerio de Educación y Ciencia (MEC) y, a su vez, como regulador local de las políticas y prácticas educativas. El AE constituye un nuevo modelo normativo de organización escolar diseñado para la operacionalización de diversas políticas socio-educativas que pasan a ser gestionadas desde la escuela donde se sitúa la 'sede' del AE. En este artículo pretendemos dar cuenta del proceso por el cual ha sido rediseñada la estructura organizacional de las escuelas agrupadas y, conjuntamente, el trabajo del Director Escolar.

\section{Transformaciones en el proceso de agrupar y dirigir escuelas en Portugal}

El proceso legislativo para regular el agrupamiento de escuelas tuvo varias etapas. En este primer apartado recuperamos algunas de las principales legislaciones que ponen en evidencia las transformaciones en los modos de regulación institucional de la política de AE. Ellas son: el DecretoLei n. $^{\circ}$ 115-A/98, de 4 de maio, que regula la conformación de los Agrupamientos Horizontales; posteriormente, el Despacho n. ${ }^{\circ} 13313 / 2003$, de 8 de julho, que promueve la creación de Agrupamientos Verticales que incluyeron escuelas desde el Jardín de Infantes hasta la Escuela Básica de Tercer Ciclo; finalmente, la Resolução do Conselho de Ministros n. ${ }^{\circ}$ 44/2010, de 14 de junho, que incluye las escuelas de enseñanza secundaria a los Agrupamiento Verticales.

En contexto de aquello que Barroso (2003b) califica como "mesianismo reformista", el Decreto-Lei n. ${ }^{\circ} 172 / 91$, de 10 de março, pone en vigor un nuevo régimen de dirección, administración y gestión de las escuelas públicas, en fase de experimentación, aprobado para ser implementado en cerca de treinta escuelas del país, pero que no llegó a ser generalizado. Este nuevo esquema se aplica a Escuelas Básicas de Segundo Ciclo, Escuelas Básicas de Tercer Ciclo (EB3), Escuelas Secundarias y en las denominadas "áreas escolares", integradas por Jardines de Infantes y Escuelas Básicas de Primer Ciclo agrupadas por zona geográfica, los cuales poseen órganos de dirección, administración y gestión comunes. De este modo se cimientan las lógicas de agrupamiento horizontales y verticales de escuelas, junto a los procesos de 
racionalización y reorganización de la red escolar.

Entre los años 1996 y 2000, el gobierno socialista del Primer Ministro António Guterres y del Ministro de Educación Marçal Grilo promueve el "Pacto Educativo para el Futuro". Se propone en primer lugar territorializar las políticas educativas y apoyar formas diversificadas de gestión, que deben servir a la adaptación a las especificidades locales a través de la celebración de contratos de autonomía entre las escuelas y el Ministerio de Educación y Ciencia (Lima, 2011). Otra de las acciones principales fue financiada por el Cuadro Comunitario de Apoyo negociado con la Comunidad Europea, creando en 1996 el Programa "Territorios Educativos de Intervención Prioritaria". En los objetivos del Programa coexisten medidas de refuerzo de la autonomía escolar y nuevas medidas de control y combate a las desigualdades escolares a través del desarrollo de programas socioeducativos orientados a la integración multicultural. Formosinho et al. (2010) describen a los Territorios Educativos de Intervención Prioritaria como un instrumento diseñado para fomentar una dimensión territorial en la autonomía de la escuela y, además, para la creación de una unidad organizacional por encima de cada escuela que agrupa a escuelas de diferentes niveles de enseñanza localizadas en proximidad geográfica.

El Despacho Normativo $n .^{\circ} 27 / 97$, de 2 de junho, dio un nuevo impulso a la creación de AE. Se refuerza la interconexión entre autonomía escolar y la racionalización de la red escolar a través de una reorganización orientada a nuevas dinámicas de asociación o agrupamiento de escuelas. Conforme a lo antes mencionado, corresponde a los órganos de administración y gestión de los niveles de enseñanza Jardín de Infante hasta la Enseñanza Básica de Tercer Ciclo el desarrollo de propuestas de asociación o agrupamiento de escuelas, así como el desarrollo de sus respectivos Proyectos Educativos y Reglamentos Internos para el ciclo lectivo 1997-1998.

En el artigo 8 del Decreto-Lei $n .^{\circ} 115-A / 98$, de 4 de maio, se opta definitivamente por la terminología de AE. En este decreto-ley un AE se define como: "una unidad organizativa dotada de sus propios órganos de administración y gestión, y constituido por establecimientos de educación preescolar y de uno o más ciclos y niveles de educación" [nuestra traducción]. Simões (2005, p. 18) reconoce, en el Decreto-Lei n. ${ }^{\circ} 115-A / 98$, el "punto de partida" decisivo para los AE. La institucionalización de los AE promueve el 
desarrollo de nuevas responsabilidades y relaciones de poder al interior y entre las escuelas. Por tanto, esta política modifica un terreno escolar carente de cualquier tipo de articulación y caracterizado por la presencia de escuelas cerradas en sus fronteras y rutinas.

Así, por primera vez, en el AE acontece la confrontación con otras posibilidades de organización escolar que cobran presencia en contexto de "crisis de soluciones" (Barroso, 2003a). Hacia 2003, el Despacho n. ${ }^{\circ} 13313$, de 8 de julho, de la Secretaria de Estado de la Administración Educativa, refuerza la apuesta del Decreto-Lei n. ${ }^{\circ} 115-A / 98$ e impone el reordenamiento de la red escolar bajo el modelo de Agrupamiento Vertical de Escuelas. Los mismos incluyen escuelas desde el Jardín de Infantes hasta la Escuela Básica de Tercer Ciclo. De este modo, queda definido el camino para la integración en un futuro, no muy lejano, de la Escuela Secundaria en los Agrupamientos Verticales. Este proceso se sustenta en una nueva reglamentación para la administración y gestión de las escuelas que presentamos a continuación.

El nuevo Modelo de Administración y Gestión de las Escuelas Públicas introducido por el Decreto-Lei $n .^{\circ} 75 / 2008$, de 22 de abril, mantiene la definición de los $A E$ como una unidad organizativa de gestión y administración. Una de las principales novedades legales es el nuevo estatuto adquirido por el Director, definido ahora como órgano unipersonal de gestión que preside, además, el Consejo Pedagógico y el Consejo Administrativo. Se pone en evidencia un cambio rotundo en la administración y gestión escolar portuguesa, signado por el retorno del Director a su condición de órgano unipersonal de gestión, esta vez en contexto de gobierno democrático.

Las profundas transformaciones en la organización y gestión de las escuelas, particularmente bajo la nueva estructura burocrática- administrativa que constituyen los $\mathrm{AE}$, ha favorecido la redefinición del trabajo de los Directores y de las condiciones organizacionales para el ejercicio de su función. Lima (2013) realiza un recorrido por las principales regulaciones para la dirección escolar, y para ello toma al año 1974 como contexto político que marca el final de la dictadura militar y promueve la transformación de la elección del Director de escuela en un proceso democrático, de naturaleza colegial. Recuperamos a continuación la cronología desarrollada por Lima (2013, p. 59): 
1. "Comisión de Gestión" entre 27 de Mayo de 1974, regulado por el Decreto-Lei n. ${ }^{\circ} 735-A / 74$;

2. "Consejo Directivo" a partir de 1974, aunque con una arquitectura formal estabilizada a través del Decreto-Lei $n .^{\circ} 769-A / 76$, que tuvo vigencia hasta 1998;

3. "Consejo Ejecutivo", a partir del Decreto-Lei n. ${ }^{\circ} 115-A / 98$, en vigencia hasta el 22 de Abril de 2008; y finalmente

4. "El Director" con la publicación del Decreto-Lei n. ${ }^{\circ} 75 / 2008$, retornando, de este modo, treinta y cuatro años después del 25 de Abril, a un órgano unipersonal, aunque electo por el ahora designado Consejo General.

A partir del nuevo Modelo de Administración y Gestión de las Escuelas Públicas el Director es reconocido como "el primer responsable a quien podrán ser atribuidas las responsabilidades por la prestación del servicio de educación pública de educación, y por la gestión de los recursos públicos puestos a su disposición" (Preámbulo del Decreto-Lei n. ${ }^{\circ}$ 75/2008, nuestra traducción). Así, la gestión unipersonal es colocada en una lógica de superioridad para ejecutar la visión del líder y del proyecto de organización que lleva adelante el Director con el apoyo de personas de su confianza, nombradas por sí mismo. El Decreto- Lei $n .^{\circ}$ 75/2008 establece, además, que el coordinador de escuela de cada institución integrada en un $A E$ debe ser nombrado por el Director de $A E$. En consecuencia, cada coordinador de escuela pasa a operar como representante del Director en su respectivo establecimiento durante los cuatros años que dura el mandato de la gestión directiva.

En la centralidad otorgada a la figura del Director como responsable de cada $\mathrm{AE}$ subyacen elementos para la erosión de la gestión escolar democrática. En este sentido, Lima (2011) sostiene que uno de los mayores obstáculos para la construcción del principio democrático de autonomía es la concentración de poderes en el Director, y la libertad que le fue concedida para seleccionar a quienes ocupan los cargos de gestión en cada escuela agrupada. En efecto, el gobierno heterónomo del AE opera a través de lo que denomina una dirección escolar atópica, o desde la Escuela Sede, un complejo aparato político-administrativo que es regulado desde las estructuras centrales y pericentrales del MEC. 
Con la dirección política del sistema educativo en el centro, pero la responsabilidad sobre la ejecución delegada en el $\mathrm{AE}$, el Director ejerce una función técnico-racional que determina la gestión del $A E$ y el trabajo de quienes fueron seleccionados para representarlo y gestionar las escuelas agrupadas.

El Agrupamiento Vertical conlleva un proceso de intensificación de las interacciones entre el MEC y el Director de AE, y será finalmente la Resolução do Conselho de Ministros $n^{\circ} 44 / 2010$, de 14 de junho, la que "culmine" el proceso de sanción de reglamentaciones que sientan las bases para la integración de la Escuela Secundaria en los Agrupamientos Verticales. Así, se establece la creación de $A E$ conformado por una escuela pública de enseñanza secundaria, la cual se constituye en Escuela Sede de AE. La Resolución mencionada establece la constitución de Agrupamientos Verticales que incluyen desde el Jardín de Infantes hasta la Escuela Secundaria. Esta política se articula a la ampliación de la obligatoriedad escolar para niños y jóvenes con edades de entre 5 y 18 años, legalmente establecida en el Decreto-Lei $n .{ }^{\circ}$ 85/2009, de 27 de agosto, que tiene por objetivo la universalización de la escolaridad hasta culminar la enseñanza secundaria.

Los principios orientadores de los Agrupamientos Verticales son: adaptar la red escolar a los objetivos de una educación obligatoria de 12 años; adecuar la dimensión y las condiciones de las escuelas a la promoción del suceso escolar y el combate del abandono escolar; y promover la racionalización de los $\mathrm{AE}$ ya existentes, con el fin de originar el desarrollo de un Proyecto Educativo común que articule los diferentes ciclos de enseñanza. La "receta indicada" para la formación de Agrupamientos Verticales establece la vigencia, hasta finales del año académico 2010/11, de escuelas no agrupadas y de Agrupamientos Horizontales compuestos exclusivamente por el mismo nivel de la educación. Así, la culminación del proceso de agrupación vertical de escuelas marca un hito que define un nuevo "paisaje" para la administración escolar portuguesa, 24 años después de la sanción de la Lei de Bases do Sistema Educativo. 


\section{La política de Agrupamiento Escolar: ¿Descentralización o desconcentración administrativa?}

La descentralización política y administrativa del Estado portugués, ya definida en la Constitución de 1976, es una palabra clave para los diferentes partidos políticos que gobernaron el país desde el retorno a la democracia. Sin embargo, en la práctica se promovió el mantenimiento de una estructura tradicional de administración centralizada y organizada por rutinas rígidas, las cuales se conservaron estables durante los últimos treinta años (Afonso, 2009). Para Barroso (2013), ni la Constitución del año 1976, ni la Ley de Bases del Sistema Educativo de 1986, por si mismas, han conseguido operar como instrumentos para modificar las bases de la gestión centralizada. Por tanto, en la descentralización se conjugan acciones promovidas con recursos y argumentos contradictorios que incluyen tanto el fomento del mercado, como así también el refuerzo de la democracia local y de la participación.

Entre los argumentos que justifican la descentralización educativa se destacan: la aproximación del lugar de decisión al de aplicación; la consideración de especificidades locales; la promoción de la participación de los utilizadores de los servicios públicos en su gestión; la reducción de la burocracia estatal; la promoción de la creatividad; y la innovación pedagógica.

Ante este panorama político-administrativo, Formosinho, Fernandes, Machado, y Ferreira (2005) manifiestan la necesidad de abordar de modo conjunto los procesos de desconcentración y descentralización. Los procesos de descentralización son generalmente precedidos de acciones de desconcentración, las cuales comúnmente se confunden o venden la desconcentración por descentralización. De este modo, la combinación de retóricas descentralizadoras con normativas que responden sólo a procesos de desconcentración o de recentralización ponen de manifiesto que la desconcentración no es necesariamente una etapa en el proceso de descentralización, todo lo contrario, puede tener por objetivo dificultarla. Los autores clarifican las definiciones conceptuales descentralización y desconcentración del siguiente modo:

Descentralización es el proceso de desarrollar tipos de administración pública menos dependientes del Estado, áreas de actividad que hasta el momento están más subordinadas a una forma más estatizada. La desconcentración es meramente un proceso de transferir a los servicios regionales y locales del Estado las competencias hasta ahí situadas en los servicios centrales; es por lo 
tanto un proceso de repartición de competencias dentro de un tipo de administración estatal directa (Formosinho et al., 2005, p. 25, nuestra traducción).

Afonso (2009) define al AE como ejemplo de transformación en la estructura de administración escolar, es decir, como promotor de aparentes mejoras en la eficiencia y en la gestión de los recursos educativos; pero que, sin embrago, acaban por promover medidas que se traducen en el cierre de escuelas pequeñas y su agrupamiento en unidades administrativas de mayores dimensiones. Asimismo, se origina el uso flexible del personal docente en virtud de la articulación entre profesionales y entre las familias y las escuelas agrupadas. Las acciones, antes mencionadas, al ser ejecutadas sin modificar las bases organizativas centralizadas del sistema educativo, hacen factible enmarcar a los AE dentro de la tipología que Formosinho et al. (2005) denominan como:

Desconcentración territorial, donde opera una administración directa del Estado a través de la creación de grados intermedios e inferiores de decisión a nivel regional o local, pero integrados en una cadena jerárquica de comando central o bien sujeto a su poder de dirección, pudiendo sus decisiones ser modificadas y revocadas por los responsables centrales (p. 27, nuestra traducción).

Es decir, en los AE algunas decisiones administrativas son tomadas por agentes en posición intermedia, aunque continúen jerárquicamente dependientes de los superiores quienes pueden alterar las decisiones. La máxima principal es que el superior determina y el inferior ejecuta, con la particularidad de que la desconcentración puede admitir que algunas decisiones administrativas sean tomadas por órganos de inferiores jerarquías, posibilitando generar respuestas más eficaces, rápidas y adecuadas a los problemas. Cuestiones que un sistema centralizado-concentrado no permite.

Lima (2011, p. 112) explica que, en su forma concentrada, los servicios centrales del MEC se constituyen en los únicos responsables por la toma de decisión. En tanto, en el modo desconcentrado, algunas decisiones importantes pueden ser tomadas por agentes en posición intermedia o inferior a la administración central entre los que existe una delegación de poder, pero sumida en una relación de obediencia. El autor afirma que cada escuela agrupada, de acuerdo con esta perspectiva, será doblemente periférica, no sólo frente a las instancias centrales y pericentrales, sino también en relación con la Escuela Sede a la que formalmente pertenece. 
La política de $A E$ y la centralidad atribuida a la Escuela Sede en la gestión escolar ha creado nuevas fronteras organizacionales. Ante este nuevo panorama político-administrativo, el desafío teórico-interpretativo a dar respuesta por la investigación desarrollada implicó el reconocimiento de las lógicas y dinámicas de acción que regulan la acción organizada, tanto entre la Escuela Sede con el MEC, como entre las escuelas agrupadas y la Escuela Sede de AE. Así, se constituye un nuevo contexto organizacional para el estudio de la dirección escolar.

\section{Notas teórico-metodológicas del estudio en el AE Margen}

Como viene siendo recuperado, en contexto de "territorialización de las políticas educativas" la figura del Director adquiere centralidad en la administración local del AE. Se espera que dirija a múltiples organizaciones escolares, ahora agrupadas, que deben compartir un mismo proyecto educativo y estar relativamente próximas en cuanto a su ubicación geográfica. Consideramos que la relevancia, especificidad y novedad de este estudio radica en la construcción de conocimiento acerca del proceso de recontextualización de la política en un $A E$ cuyos procesos organizativos internos han alcanzado cierto nivel de consolidación en el tiempo.

A partir de las contribuciones de la teoría de la Regulación Social de Reynaud (1997, 2003), en esta investigación procuramos abordar, desde planos complementarios, los mecanismos de regulación institucional que la política de $\mathrm{AE}$ impone al Director, y los mecanismos de regulación institucional y situacional que el Director ejerce sobre los actores que contribuyen a la gestión del AE. En el presente estudio consideramos, además, los aportes de la Sociología de la Acción (Crozier \& Friedberg, 1977; Friedberg, 1995) y principalmente de las categorías de Acción Organizada y Sistema de Acción Concreto (SAC). Esta opción teórica, y también metodológica, tiene por objetivo desnaturalizar la figura normativa de los AE para comprender esta nueva configuración organizacional desde el accionar de los actores y las lógicas de acción que legitiman conjuntamente.

El desarrollo de esta investigación se contextualiza en un Agrupamiento Vertical, perteneciente a la Dirección Regional de Lisboa y Valle del Tejo. EI AE se conforma por doce escuelas, específicamente es el 
resultado de la fusión de dos Agrupamientos Horizontales y de tres escuelas no-agrupadas, siendo la Escuela Sede la Escuela Secundaria. A fin de resguardar la identidad del $\mathrm{AE}$ en estudio, y de los actores involucrados, optamos por otorgarle una denominación ficticia: AE Margen.

EI SAC en análisis se integra por la Directora y un conjunto específico de actores que contribuyen a la gestión del AE por iniciativa y elección directa de la Directora, los cuales son denominados como 'actores escolares'. Los 'actores escolares' que conforman el SAC estudiado son: la Directora del AE; el Equipo Directivo: una Sub-Directora y tres Adjuntas de Dirección; tres Coordinadoras de las Estructuras de Coordinación Intermedia y Supervisión Pedagógica, denominadas por los propios 'actores escolares' como "Estructuras Intermedias de Gestión": la Coordinadora del Gabinete de Inclusión Ciudadana, la Coordinadora del Gabinete de Evaluación Interna y la Coordinadora del Departamento Curricular de Educación Básica. Se incluyen además los siete Coordinadores de Escuela y cuatro Interlocutores, que se desempeñan en las once escuelas agrupadas del $\mathrm{AE}^{2}$.

El objetivo general que guía este estudio se define del siguiente modo: describir, analizar e interpretar el Sistema de Acción Concreto construido en la interacción entre la Directora del $\mathrm{AE}$, su Equipo Directivo, las Estructuras Intermedias de Gestión, los Coordinadores de Escuela e Interlocutores. Específicamente, se pretende describir, analizar e interpretar: los procesos organizativos internos del SAC (re)creados por los 'actores escolares' del AE; las lógicas de acción regulatorias legitimadas en el SAC por los 'actores escolares' del AE; y los efectos del SAC en los 'actores escolares' del AE.

El diseño de la investigación es cualitativo y responde a un estudio de caso intrínseco, opción metodológica seleccionada para abordar las especificidades del SAC. En las elecciones teórico-metodológicas de este estudio se articula el registro interpretativo con un enfoque inductivo, a partir del cual se pretende interpretar la acción organizada, complementando un enfoque inductivo con un análisis deductivo (Friedberg, 1995).

El trabajo de campo de esta investigación se desarrolló durante el ciclo lectivo 2012-2013. Se realizaró un total de veinte entrevistas semiestructuradas a los 'actores escolares' elegidos por la relevancia de las funciones que desempeñan en la gestión del $\mathrm{AE}$, como así también en la promoción de la articulación entre la Escuela Sede y las escuelas agrupadas. 
Estas entrevistas permitieron la recolección y posterior análisis, a través de procedimientos de análisis de contenido de los datos significativos para la interpretación del SAC contextualizado en el AE Margen.

\section{Nuevo contexto para la acción organizada en AE: ¿Nuevo paisaje para la dirección escolar portuguesa?}

El estudio de caso en el AE Margen permite deducir que el AE, como nuevo modelo normativo de organización escolar, ha promovido que la Directora del AE construya el vínculo y el control sobre las escuelas agrupadas, principalmente a través de las Estructuras Intermedias de Gestión y del Equipo Directivo. En consecuencia, consideramos que existe una especie de "actualización" de la relación que Barroso (1991) planteaba para caracterizar el vínculo construido entre los Directores y los profesores, cuyo sentido era compatibilizar las soluciones administrativas y el ejercicio de las exigencias pedagógicas del acto educativo. La reformulación promovida por la política de $\mathrm{AE}$ implica reconocer la existencia de un nuevo contexto de acción organizada a través del cual la Directora gestiona el AE: la zona de acción de la Directora del AE se articula con la zona de los Coordinadores de Escuela a través de las Estructuras Intermedias de Gestión y del Equipo Directivo. En estas dos estructuras la Directora del $A E$ desconcentra responsabilidades que compatibilizan el control sobre la gestión del AE, con las exigencias pedagógicas del acto educativo y las respuestas administrativas que demandan las escuelas agrupadas.

La Directora del AE Margen ha gestionado una red de relaciones que sirven a la gestión de una "arquitectura de acuerdos" que posibilita la adaptación a las múltiples regulaciones que se adicionaron a la Escuela Sede para la gestión del AE Margen o de doce escuelas al mismo tiempo.

Desde los aportes de Barroso (2011), reconstruir la red de relaciones gestionada por la Directora del AE Margen supone reconocer, en primer lugar, "su" asimilación a un órgano desconcentrado del MEC, que actúa en nombre de la administración central en el AE. En segundo lugar, "su" definición como órgano de gestión unipersonal que desconcentra responsabilidades en las Estructuras Intermedias de Gestión del AE y en el Equipo Directivo. En tercer lugar, "su" reconocimiento como órgano descentralizado y representante de la 
gestión local del AE, que opera por medio de los órganos de gestión escolar que preside y a través de los cuales promueve finalmente, en cuarto lugar, la recentralización de la gestión desconcentrada a las Estructuras Intermedias de Gestión y al Equipo Directivo. Importa destacar que la Directora, más allá de ser un órgano unipersonal de gestión, y una delegada del poder central, responde también ante el Consejo General de AE, pudiendo este dimitirla. De esta forma lo expresa la Directora:

Siempre estoy yo (risas) en el Consejo Administrativo, somos tres: yo, la Adjunta y el jefe de servicios administrativos. En el Consejo Pedagógico están los Coordinadores, y por ley soy yo quien lo preside, y en Consejo General estoy presente para resolver dudas pero sin derecho a voto. (Directora del AE Margen)

Las nuevas estructuras de administración y gestión escolar del AE Margen posibilitan hipotetizar sobre la conformación de un "nuevo paisaje para la gestión escolar portuguesa"3. En el caso del AE Margen hablamos concretamente de una nueva estructura burocrático-administrativa a través de la cual la Directora dirige el $\mathrm{AE}$, con base en un conjunto de potestades que, como órgano unipersonal de gestión, posee para seleccionar a los 'actores escolares', distribuir funciones y definir, de este modo, una "arquitectura" para gestionar el $\mathrm{AE}$.

Al parecer, la Directora del AE Margen está consiguiendo poner en funcionamiento "el montaje arquitectónico" cimentado en procesos organizativos internos y en lógicas de acción regulatorias funcionales a la gestión a distancia de las escuelas agrupadas y al centralismo de la Escuela Sede del AE. Referir a esta arquitectura y a su buen funcionamiento no significa hablar de ausencia de conflictos; los problemas que acontecen en las diferentes esferas de gestión acaban por ser resueltos conforme a los recursos disponibles, al voluntariado y a la responsabilidad asumida por los Coordinadores de Escuela e Interlocutores. Son aspectos que ponen en evidencia el compromiso asumido, por parte de los actores escolares para con la Directora del AE que los seleccionó, como así también implica la aceptación de las condiciones objetivas disponibles para gestionar las escuelas agrupadas.

En el análisis efectuado emerge la centralidad de la Directora del AE Margen en la regulación de la "arquitectura de acuerdos" que posibilita la 
gestión del AE, desde la Escuela Sede y a través del SAC que diseñó. El refuerzo de la capacidad reguladora de la Directora, a través del caudal de competencias adquiridas para la regulación interna del AE Margen, da cuenta del ejercicio de poder del MEC por medio de la creación de condiciones organizacionales para re-regular el trabajo del Director - una figura que junto a los vaivenes de la política portuguesa ha mudado numerosas veces en su denominación legal y en sus potestades.

\section{Avances interpretativos. Mutaciones en la dirección y en la autonomía escolar}

Los resultados de la investigación posibilitan reconocer que el SAC conformado por los 'actores escolares' del AE Margen para recontextualizar la política de AE está dando respuestas a las demandas ejecutivas que el MEC ha desconcentrado en la Directora del AE Margen. Frente a esta aseveración se torna necesario definir a la Directora del AE como un 'regulador técnico' de la arquitectura de gestión del $\mathrm{AE}$. Es ella quien gestiona, eficientemente, la desconcentración de funciones en su "equipo de confianza". Por lo tanto, es posible reconocer que la Directora cumple una función operacional de regulación interna del $\mathrm{AE}$ que se articula a la representación del MEC en la Escuela Sede.

La desconcentración de escuelas que el MEC re-centralizó en la figura del AE dirigido por un Director propicia el diseño del territorio escolar para que opere la balanza que, para Barroso (2013), posibilita el "equilibrio entre centralización y descentralización". A través del Equipo Directivo y de las Estructuras Intermedias de Gestión, la Directora del AE Margen estaría efectuando un "peligroso balance" que sostiene el "débil equilibrio" entre las escuelas agrupadas y el MEC. Es decir, por medio del trabajo de la Directora, el MEC consigue re-centralizar el control sobre el sistema educativo descentralizado-desconcentrado.

En cada uno de 'los extremos' del SAC operan la Directora del AE y los Coordinadores de Escuela. El extremo de los Coordinadores de Escuela e Interlocutores del AE Margen se caracteriza por la presencia mayoritaria de Escuelas Básicas de Primer Ciclo y a Jardines de Infantes. Estos actores son los representantes de la Directora en las escuelas agrupadas, es decir, son 
traductores, trasmisores y ejecutores de las directrices definidas en la Escuela Sede. Se puede referir a ellos, metafóricamente, como 'satélites' que detectan conflictos y actúan para resolverlos con el apoyo de las Adjuntas de Dirección. El trabajo de campo desarrollado nos condujo a interrogarnos por el sentido, el "¿para qué?" de los Coordinadores de Escuela e Interlocutores en el AE. Es pertinente introducir y aclarar que ellos se auto-reconocen, en su mayoría, como actores carentes de autonomía en el ejercicio de sus funciones. Fue posible registrar en las entrevistas, incluso, el "no-deseo de autonomía" y, por ende, de responsabilidad sobre las acciones que ejecutan. Consideramos que estos actores cumplen una función que los transforma en garantes del orden y del funcionamiento escolar, constituyéndose, además, en transmisores de una figura de autoridad para los docentes, padres, alumnos, no-docentes.

Dado que la mayor parte de las escuelas agrupadas son Escuela Básicas de Primer Ciclo y Jardines de Infantes, es oportuno recuperar los aportes de la investigación desarrollada por Dias (2006) en relación al trabajo que desempeñan los Directores del nivel primario de enseñanza. La autora recupera investigaciones de la década del noventa que ya referían a los escasos márgenes de decisión y a las múltiples dependencias jerárquicas que la gestión del nivel poseía en el contexto en que desarrollaron las investigaciones. Dias (2006) clarifica que, en el periodo anterior a la implementación del Decreto-Lei n. ${ }^{\circ} 115-A / 98$, la diferenciación funcional entre el trabajo de los Directores y los profesores no era sentida por los docentes como una diferencia de estatuto o de autoridad, sino como una forma cómoda por la cual los profesores evitaban desarrollar tareas no deseadas, como las administrativas.

Sin pretender comparar la función del Coordinador de Escuela con la del "antiguo" Director de Escuela Primaria, el contraste entre las investigaciones citadas posibilita deducir que este nivel de enseñanza posee ya una trayectoria bajo un modelo de gestión estatal, principalmente burocrático-administrativo. Es necesario acrecentar que el Agrupamiento Vertical de escuelas podría representar una nueva dependencia de estos niveles de enseñanza a las lógicas de gestión de la Escuela Secundaria, ahora Escuela Sede de AE.

En las escasas trayectorias profesionales de Coordinadores de Escuela e Interlocutores en cargos directivos, encontramos un factor que 
propicia las dinámicas de recentralización del control sobre las escuelas agrupadas, haciendo que sean aceptadas y naturalizadas. Por tanto estos "nuevos actores escolares" (Coordinadores de Escuela, en algunos casos profesores sin curso a cargo en el momento que fue desarrollado el trabajo de campo), a partir del apoyo que reciben del Equipo Directivo para gestionar las escuelas agrupadas, también construyen su legitimación y una nueva identidad en las funciones que desempeñan en el $\mathrm{AE}$ : la de representantes de la Directora del AE.

En el otro extremo del continuum analizado esta la Directora. El trabajo que desarrolla se caracteriza por la confluencia de tres funciones: la primera, asociada a la supervisión y acompañamiento del trabajo del Equipo Directivo, como así también de las Estructuras Intermedias de Gestión; la segunda, vinculada a la promoción y planificación de las relaciones interinstitucionales que se gestionan desde el Gabinete de Inclusión Ciudadana; la tercera, centralizada en el trabajo burocrático por el cual rinde cuentas a las múltiples instancias evaluadoras del MEC.

Para la regulación interna del SAC del AE Margen, la Directora del AE y los Coordinadores de Escuela e Interlocutores han asumido nuevas funciones que, si bien implican una relación jerárquica, poseen como rasgo común una declarada "carencia de autonomía". Como ya fue referido, en el caso de los Coordinadores de Escuela e Interlocutores, esta carencia se asocia a la consulta y la transmisión de información a la Escuela Sede, respecto de las decisiones que toman. De un modo similar, la autonomía de la Directora se reduce a una "autonomía para pedir autorizaciones", una expresión por ella utilizada. En las referencias acerca de la autonomía escolar es factible reconocer que ambas realizan un uso retórico del discurso de autonomía escolar, que es consecuente a la reconfiguración de las relaciones de poder en un nuevo modelo normativo de organización escolar. De esta manera, bajo la apariencia de distribuir y descentralizar el poder de decisión, se refuerza el centralismo del MEC y se transforma la autonomía escolar en un vocablo necesario, pero vacío de sentido en las diferentes instancias de gestión del AE.

EI SAC contextualizado en el AE Margen posibilita pensar que al aceptar los márgenes de autonomía relativa que poseen los Coordinadores de Escuela y la Directora del AE, también se legitiman nuevas relaciones de 
poder y nuevos niveles jerárquicos entre las escuelas agrupadas y la Escuela Sede y, a su vez, entre la Escuela Sede y el MEC.

\section{A modo de cierre. Sobre la "muerte" del Director escolar: hacia una nueva regulación territorial e intermedia}

Es posible reconocer que la complejidad organizacional del $A E$ se constituye en un instrumento que desencadena una reconfiguración de la identidad del Director. El Director se transforma en una figura que, a pesar de mantenerse presente y de ser cada vez más relevante en los textos políticos, en el contexto de la práctica (Ball, Maguire, Braum, Hoskins, \& Perryman, 2012) de la administración escolar ve las especificidades que caracterizaron a su función siendo redefinidas.

Las características del trabajo que desempeña la Directora en el AE Margen conllevan a que se constituya en un "regulador territorial" del conjunto de escuelas agrupadas. La concepción de Director escolar como un líder profesional (Barroso, 2005, p. 429) no desaparece del imaginario de la Directora. Sin embargo, como ya fue referido, en la práctica las dimensiones del $\mathrm{AE}$ hacen que tales funciones sean ejecutadas a través del Equipo Directivo, responsable del vínculo con las escuelas agrupadas. Es posible considerar, inclusive, que estos actores también promueven un 'liderazgotécnico; ello significa que, pese a tener un vínculo más próximo con los Coordinadores de Escuela, sus tareas son, de igual modo, ejecutadas a distancia (desde la Escuela Sede) y a través de las redes de comunicación con las escuelas agrupadas.

Sería importante analizar, en una futura investigación, las influencias que la arquitectura de acuerdos construida por la Directora de AE ejerce en los modos de enseñar y evaluar de los profesores, siendo posible hipotetizar que esta fórmula organizacional podría estar limitando o inclusive impidiendo el liderazgo pedagógico del equipo directivo de $\mathrm{AE}$.

El análisis sobre el trabajo que desempeña la Directora del AE posibilita inferir que hay un "viejo actor escolar" ausente en la gestión del AE: el Director Escolar. Dada su función de "regulador territorial", la Directora del $\mathrm{AE}$ ya no es más Directora de la Escuela Secundaria, cargo que desempeñó hasta la conformación del AE Margen. Concretamente asistimos a la 
desaparición de la figura del Director como representante de una unidad organizacional, para pasar a hablar de escuelas direccionadas centralmente a distancia en la Escuela Sede, o en la nueva sede administrativa desconcentrada del MEC.

En el plano de las orientaciones para la acción que regulan el trabajo de la Directora, en la auto-imagen que ella construye sobre su labor, como así también en la imagen que el conjunto de actores escolares poseen, es posible identificar transformaciones en la dirección escolar. Seguramente la prolongación de este trabajo de campo posibilitará identificar que queda en la práctica de aquella Directora de Escuela Secundaria devenida en Directora de $\mathrm{AE}$.

Es evidente el cambio en la estructura burocrático-administrativa por la cual la Directora ejerce su función; pero además, parecen ser irreversibles las transformaciones en el trabajo que el MEC demanda para dirigir un AE. Los resultados de la investigación "invitan" a continuar la indagación sobre los sentidos de la "muerte del trabajo de Director" en el contexto de la práctica escolar. En su reemplazo emerge un nuevo actor: el Director de $\mathrm{AE}$, como 'regulador intermedio', regulado por el MEC y regulador territorial de las prácticas que acontecen en un complejo SAC. Un actor que, formalmente, aún no aparece en las legislaciones, pero encarna en las tareas a las que debe responder el management de una "nueva empresa educativa".

Investigar el Sistema de Acción Concreto contextualizado en el $A E$ Margen supuso, desde luego, investigar las traducciones situacionalmente construidas para poner en acto la política de $\mathrm{AE}$, lo que demanda devolver a los actores escolares la centralidad que poseen en las políticas educativas, como texto y contexto, y como sujetos y objetos de la reorganización del Sistema Educativo portugués.

\section{Agradecimientos}

Gracias al Programa Move on Education, Erasmus Mundus de la Comisión Europea por la beca otorgada y por haber hecho posible esta valiosa oportunidad profesional. Gracias al Profesor Dr. Luís Miguel Carvalho por el compromiso asumido para con mi formación y la orientación de esta investigación. Gracias a los actores escolares del Agrupamiento Escolar Margen por la apertura institucional y el apoyo que me brindaron durante el desarrollo de la investigación. 


\section{Notas}

1 Tesis doctoral titulada "El Director de un Agrupamiento Escolar. Regulado y regulador de las políticas educativas", dirigida por el Dr. Luis Miguel Carvalho. Doctorado en Educación, Especialidad en Política y Administración Escolar, Universidad de Lisboa. Tesis aprobada en marzo de 2014.

2 En el trabajo de campo fue posible conocer la figura del Interlocutor. Se trata de un 'actor escolar' creado por la Directora del AE para dar respuesta a la imposibilidad legal de nombrar coordinadores de escuela en instituciones con menos de tres profesores en ejercicio efectivo de la función docente. El Interlocutor es el representante de la Directora en estas pequeñas escuelas EB1 o Jl. La asignación de este cargo es consecuencia de una valoración positiva y simbólica de la labor de los profesores que no implica incremento salarial y que, desde luego, no está regulada en ningún estatuto.

3 Paráfrasis de la idea que Dussel, Birgin, y Tiramonti (2001) desarrollan para caracterizar el Sistema Educativo argentino posterior a la sanción de la Ley de Transferencia Educativa en el año 1991.

\section{Referencias}

Afonso, N. (2005). Prefácio. In G. Simões, Urdindo a teia: As linhas e lógicas de acção na emergência e construção do Agrupamento "vertical" de Escolas de Figueiró dos Vinhos (pp. 9-11). Lisboa: Universidade de Lisboa.

Afonso, N. (2009). A direção das escolas públicas em Portugal: Dinâmicas do contexto e lógicas de acção dos gestores escolares. In G. Barzanò, Culturas de liderança e lógicas de responsabilidade. As experiências de Inglaterra, Itália e Portugal (pp. 15-24). Vila Nova da Gaia: Fundação Manuel Leão.

Ball, S., Maguire, M., Braum, A., Hoskins, K., \& Perryman, J. (2012). How schools do policy. Policy enactments in secondary schools. New York: Routledge.

Barroso, J. (1991). Modos de organização pedagógica e processos de gestão da escola: Sentido de uma evolução. Inovação, 4, 55-86.

Barroso, J. (2003a). A escola pública: Regulação, desregulação, privatização. Porto: ASA.

Barroso, J. (2003b). Organização e regulação dos ensinos básico e secundário, em Portugal: Sentidos de uma evolução. Educação e Sociedade, 24(82), 63-92.

Barroso, J. (2005). Liderazgo y autonomía de los centros educativos. Revista Española de Pedagogía, LXIII(232), 423-441.

Barroso, J. (2011). Direcção de escolas e regulação das políticas: Em busca do unicórnio. In J. Costa \& A. Neto-Mendes, A emergência do director da escola: Questões políticas e organizacionais (pp. 11-22). Aveiro: Universidade de Aveiro.

Barroso, J. (2013). A emergência do local e os novos modos de regulação das políticas educativas. Educação - Temas e Problemas, 12 e 13, 13-27. 
Crozier, M., \& Friedberg, E. (1977). L'acteur et le système. Paris: Seuil.

Dias, M. (2006). As escolas primárias portuguesas na viragem do século: Actores e processos de mudança (1986-2003). Administração Educacional. Revista do Fórum Português da Administração Educacional, 6, 28-36.

Dussel, I., Birgin, A., \& Tiramonti, G. (2001). Hacia una nueva cartografía de la reforma curricular. Reflexiones a partir de la descentralización educativa argentina. En G. Tiramonti, Modernización educativa en los 9 (pp. 132-161). Buenos Aires: FLACSO.

Formosinho, J., Sousa Fernandes, A., Machado, J., \& Ferreira, H. (2010). Autonomía da Escola Pública em Portugal. Vila Nova da Gaia: Fundação Manuel Leão.

Formosinho, J., Fernandes, A., Machado, J., \& Ferreira, F. (2005). Administração da educação. Lógicas burocráticas e lógicas de mediação. Porto: Edições ASA.

Friedberg, E. (1995). O poder e a regra. Dinâmicas da acção organizada. Lisboa: Instituto Piaget.

Lima, L. (2011). Administração escolar: Estudos. Porto: Porto Editora.

Lima, L. (2013). Diretor(a) de escola pública: Unipessoalidade e concentração do poder no quadro de uma relação subordinada. In V. Peroni (Org.), Redefinições das fronteiras entre o público e o privado: Implicações para a democratização da educação (pp. 58-81). Brasília: Liver Livro.

Reynaud, J.-D. (1997). Les règles du jeu. L'action collective et la régulation sociale. Paris: Armand Colin.

Reynaud J.-D. (2003). Régulation de contrôle, régulation autonome, régulation conjointe. In G. de Terssac (dir.), La théorie de la régulation sociale de JeanDaniel Reynaud (pp. 103-113). Paris : La Découverte.

Simões, G. (2005). Urdindo a teia: as linhas e lógicas de acção na emergência e construção do Agrupamento "vertical" de Escolas de Figueiró dos Vinhos. Lisboa: Universidade de Lisboa.

\section{Legislación}

Decreto-Lei n. ${ }^{\circ} 115-\mathrm{A} / 98$, de 4 de maio

Decreto-Lei $n .{ }^{\circ} 85 / 2009$, de 27 de agosto

Decreto-Lei ${ }^{\circ}{ }^{75 / 2008}$, de 22 de abril

Despacho $n^{\circ} 13313 / 2003$, de 8 de julho

Despacho Normativo n. ${ }^{\circ} 27 / 97$, de 2 de junho

Resolução do Conselho de Ministros n. ${ }^{\circ} 44 / 2010$, de 14 de junho 


\section{POLÍTICA dE AGRUPAMENTO dE ESCOLAS: MUTAÇÃO OU "MORTE" DO TRABALHO DO DIRETOR DE ESCOLA?}

Resumo

A política de Agrupamento Escolar (AE) definiu um novo modelo normativo de organização escolar em Portugal. Através deste artigo, enquadrado numa tese de doutoramento, procuramos explicar (a) o processo histórico até à configuração atual da política de AE. Seguidamente, abordamos (b) as implicações do AE como uma política que, discursivamente, tem como objetivo promover a descentralização do Sistema Educativo português. Posteriormente, apresentamos (c) os principais eixos teóricos e metodológicos do estudo de caso desenvolvido num $\mathrm{AE}$ pertencente à Direção Regional de Lisboa e Vale do Tejo e (d) alguns dos principais resultados obtidos na investigação. Finalmente, são indicados (e) alguns avanços interpretativos a respeito das mudanças na direção escolar do ficcionalmente chamado "AE Margem", especificamente no trabalho do seu Diretor, uma figura que já não voltará a coordenar e dirigir apenas uma escola.

Palavras-chave

Políticas educativas; Agrupamento Escolar; Diretor de Agrupamento Escolar

\section{SCHOOL CLUSTER POLICY: MUTATION OR "DEATH" OF THE PRINCIPAL'S WORK?}

Abstract

The School Cluster (SC) policy defined a new regulatory model of school organization in Portugal. Throughout this article, based on a doctoral thesis, we try to: (a) account for the historical process until the establishment of the current SC policy; (b) address the implications of SC as a policy that, discursively, aims to promote decentralization of the Portuguese educational 
system. Afterward, we introduce (c) the main theoretical and methodological structure of the case study developed in a SC belonging to the Regional Directorate of Lisboa and Vale do Tejo and (d) we present some results obtained in the investigation. Finally, (e) we point out some of the main interpretive developments regarding the changes in school management of the fictionally called "SC Border", specifically in the position of its Director, an actor that is not going to return to the coordination and management of a single school.

Keywords

Educational policies; School Cluster; School Cluster Principal

Recebido em março/2015 Aceite para publicação em fevereiro/2016

i Facultad de Filosofía y Humanidades, Universidad Nacional de Córdoba, Argentina.

Toda a correspondência relativa a este artigo deve ser enviada para: María Cecilia Bocchio, Obispo Fajardo 1881 Barrio: Ayacucho, Córdoba, Argentina CP: 5001. E-mail: mcbocchio@gmail.com 